\title{
Early career researchers' perspectives and roles in patient-oriented research
}

\author{
Geneviève Rouleau ${ }^{1,2^{*}}$ (D), Jean-Christophe Bélisle-Pipon ${ }^{3,4}$, Stanislav Birko ${ }^{5,6}$, Philippe Karazivan 1,7, \\ Nicolas Fernandez ${ }^{7}$, Karine Bilodeau $^{8}$, Yi-Sheng Chao ${ }^{9}$, Alexandra de Pokomandy ${ }^{10}$, Véronique Foley ${ }^{11}$, \\ Bruno Gagnon ${ }^{12}$, Samantha Gontijo Guerra ${ }^{13}$, Cynthia Khanji1 ${ }^{14}$, Catherine Lamoureux-Lamarche ${ }^{13}$, \\ Bertrand Lebouché ${ }^{10}$, Carlotta Lunghi ${ }^{15}$, Matthew Menear ${ }^{16}$, Bruno D. Riverin ${ }^{17}$ and Claudie Rodrigue ${ }^{14}$
}

\begin{abstract}
Plain English summary
Patient-oriented research (POR) has received increasing attention in recent years. In this approach, patients' experiential knowledge, derived from their experiences of living with a condition or illness and of interacting with the healthcare system, is recognized, valued, and seen as complementary to scientific knowledge. Early career researchers (ECRs) are the next generation of researchers, but little is known about how they perceive POR. In this study, ECRs were invited to reflect on what POR is, how patients can best contribute to research, and ECRs' own role in developing POR. Using a technique designed to collect expert opinions and find consensus - the Delphi method - a panel of 16 ECRs responded, in three rounds, to three questionnaires, with the second and third being built on responses to the preceding ones. Based on their understanding, the panelists agreed that the most important element in defining POR would be valuing, mobilizing, and legitimizing the experiential knowledge of patients who live with a particular health condition. Panelists considered patients to be integral members of the research team, but were less convinced that they should be considered co-researchers. The panelists saw themselves as taking part in developing POR by sharing information, teaching, and encouraging POR among their peers, as well as by participating actively in organizations interested in POR. This is the first study to examine the perspectives of ECRs, who, along with many others, have an important role in supporting the on-going development of POR so that it becomes more widely adopted.
\end{abstract}

\section{Abstract}

Background Literature on patient-oriented research (POR) is growing rapidly. This field is increasingly encouraged by funders and structured by new research networks. POR involves moving away from considering patients as 'subjects', towards perceiving them as experts with experience-based knowledge. However, little is known about how early-career researchers (ECRs) perceive POR and their roles in developing it. This study examined how ECRs perceive POR, patients' roles, the future of POR, and their own role in developing this approach.

Methods A three-round Delphi study was conducted with Quebec's Strategy for People and Patient-Oriented Research and Trials (SUPPORT) Unit awardees, composed of graduate students and clinicians, all ECRs. Of the 25 invited, 18 agreed to participate (72\%), with a three-round retention rate of $89 \%$ ( $n=16$ on 18). Panelists answered (Continued on next page)

\footnotetext{
* Correspondence: genevieve.rouleau.chum@ssss.gouv.qc.ca

${ }^{1}$ University of Montreal Hospital Research Centre, Montreal, Quebec H2X0A9,

Canada

${ }^{2}$ Faculty of Nursing, Laval University, Quebec, Canada

Full list of author information is available at the end of the article
}

(c) The Author(s). 2018 Open Access This article is distributed under the terms of the Creative Commons Attribution 4.0 International License (http://creativecommons.org/licenses/by/4.0/), which permits unrestricted use, distribution, and reproduction in any medium, provided you give appropriate credit to the original author(s) and the source, provide a link to the Creative Commons license, and indicate if changes were made. The Creative Commons Public Domain Dedication waiver (http://creativecommons.org/publicdomain/zero/1.0/) applies to the data made available in this article, unless otherwise stated. 
(Continued from previous page)

open-ended questions, selected the most salient statements, and rated their (dis)agreement with proposals using a 7-point scale.

Results Five main themes emerged: 1) ECRs' knowledge of and experience with POR; 2) the POR definition; 3) patients' roles and contributions; 4) the future of POR; and 5) ECRs' roles in POR development. This study revealed that the ECRs were not so familiar with POR, even given their opportunities for networking within a scholarship program. Panelists agreed on the main components of a POR definition: valuing, mobilizing, and legitimizing the experiential knowledge of patients living with a health condition; conducting research that focuses on patients' concerns, participation, and outcomes; and integrating active partnership among a variety of actors. Panelists considered patients to be integral members of the research team $(M=5.31 \pm 1.66)$, but were less convinced they should be considered co-researchers $(M=4.50 \pm 1.75)$. Panelists saw themselves as playing many roles in developing POR, such as becoming well-informed about it and acting as knowledge brokers, motivators, doers, delegators, and activists.

Conclusion The ECRs' perspectives are informative on how the next generation of researchers envision POR, its future and how they might contribute to developing this approach. There is a clear need for a coherent and concerted strategy for POR capacity development, in which ECRs' perspectives and their specific needs are taken into account.

Keywords: Patient engagement, Patient-oriented research, Patient outcomes research, Early-career researcher, Graduate student, Health sciences research, Delphi, Academic training

\section{Background}

There is a growing body of literature on patient-oriented research (POR). The Canadian Institute of Health Research (CIHR) [1, 2] defines POR largely as a continuum of research conducted with multidisciplinary teams that engages patients as partners (as well as other relevant stakeholders), focusses on patient-identified priorities and is intended to improve patient outcomes, healthcare systems and practices. Traditionally, patients' involvement in research (e.g. clinical trials) has been limited to the role of research 'subjects'. However, the research community is beginning to move away from seeing patients as subjects and towards recognizing them more as 'experts' [3]. Considering patients as experts is a departure from the previous dominant paradigm in which knowledge resides within the medical professions and academia, while the patient plays a passive role [4]. POR is grounded in the fundamental principle of valuing patients' experience-based knowledge and mobilizing it to support health research $[5,6]$. Experience-based expertise has been defined by Duffett as "the skills and knowledge that is derived from personal experiences, such as living with a chronic illness and coping with the daily management required, and its impact on one's life" (p. 114) [7]. Recognizing experiential expertise thus implies that patients' narratives and knowledge contribute to shaping the research process.

Research funding agencies around the world now support and encourage POR. For example, in 1996, the National Institute for Health Research (NIHR) established the INVOLVE initiative to encourage active public involvement in public health and social care research [8]. Since 2010, the Patient-Centered Outcomes Research Institute (PCORI) in the United States has provided 2.2 billion dollars in funding to promote comparative effectiveness research conducted in partnership with patients [9]. In Canada, the federal government has opened up research funding opportunities through the CIHR Strategy for Patient-Oriented Research (SPOR) program. SPOR is a ten-year national strategy with five core components: SPOR networks, SPOR Support for People and Patient-Oriented Research and Trials (SUPPORT) Units, clinical trials initiatives, capacity development, and patient engagement [1]. In April 2016, CIHR invested \$357 million for SPOR [10], demonstrating Canada's commitment to that program. These considerable and important national and international initiatives were intended to transform how research is done in many countries, including Canada. This could potentially have an impact on the next generation of researchers, who are today beginning their research careers. It is thus essential to understand how early-career researchers (ECRs, i.e. trainees and untenured researchers [11]) perceive POR.

The POR literature to date has targeted a wide range of topics and questions, such as: POR's definitional and operational frameworks (still under debate) [12, 13], patients' roles and responsibilities, research activities in which they might or should be involved, their levels of involvement, at what times they should be involved [1417]. A growing body of literature carries on mapping 
and evaluating impacts of POR (targeting mainly patient engagement/public involvement) on various types of outcomes such as: key stakeholders (e.g. service users/ patients, researchers, community), consequences of POR (e.g. effectiveness, research validity) and the process of engagement itself [18-21]. Despite such interest and impact on research outcomes (e.g. increasing participant enrolment, decreasing attrition, setting research priorities, enhancing dissemination of study findings), there is a lack of robust evidence demonstrating the extent to which POR affects patient outcomes, healthcare access, cost-effectiveness, etc. [21, 22]. The purpose of this study is not intended to fill this gap, but we acknowledge this body of literature in ECRs' inquiry of POR.

Despite growing attention to POR in the research community, little is known about ECRs' perceptions of and attitudes towards it. ECRs find themselves at a point in their career where research development is expected of them [23]. As Nicholas et al. [24] envision: "Representing as they do the 'new wave' of researchers (...) ECRs constitute the breeding ground for tomorrow's established researchers" (p. 157). Their development of POR-related skills, acuity, and expertise will help foster the new research paradigm of meaningful POR. To support ECRs in this transition to fully independent research with positive roles in advancing POR approaches, it is important to understand how they perceive POR and their roles in developing, cultivating, and sustaining this approach.

This study's primary aim was twofold: 1) examine ECRs' perceptions and roles regarding POR; 2) allow to come to light recommendations favoring POR by ECRs. This study's secondary aim was to examine ECRs' perceptions of the ethical dimensions of POR [25]. The focus of the present paper is to meet the first objective, by obtaining insights into ECRs' perceptions of how POR should be defined, its purposes, patients' roles and contributions, the anticipated future of POR, and ECRs' roles in promoting POR development and deployment.

\section{Methods \\ Materials and methods Study design}

A Delphi survey was conducted with a panel of ECRs involved in POR in the Canadian province of Quebec. The study's three rounds were conducted from April to November 2016 using the Limesurvey web-based platform, from which emails were sent inviting panelists to each round. Ethics approval was obtained through the Health Research Ethics Committee (CERES) at the University of Montreal (\#16-044-CERES-D). Online informed consent was obtained from all participants.
The Delphi methodology, which uses iterative controlled feedback to elicit the opinions and comments of a panel of experts, was chosen because it produces structured, confidential, and asynchronous group communication and thereby avoids the drawbacks of non-anonymous group communications [26, 27]. In particular, due to the as-yet uncertain nature of how regulatory environments may affect the practice of POR, the policy Delphi approach was employed, "because it can address a multiplicity of issues and provide direction for policy changes" ([28], p. 308, [29]). This approach, while driven by casting a wide net in order to capture the greatest diversity of viewpoints, still allows for identifying areas of (differing levels of) agreement among expert communities. Research guidelines [30-32] have been useful to guide the conduct of the Delphi study, especially, in structuring the first round, defining the sample expertise, collecting the data and applying ethical considerations. Considering that the two principal investigators were also research participants, the presence of the third researcher allowed to preserve a degree of panelist anonymity and ensure the Delphi process was led by all panelists' views. The Delphi aimed at identifying the main trends while maintaining the diversity of perspectives that form the richness of the results. The Delphi study flowchart is presented in Fig. 1, with the timeline of the three rounds and the main topics covered.

Three rounds were conducted: (1) a 'classic' Delphi first round, consisting of open-ended questions regarding the definition, feasibility, and utility of POR, and a few demographic questions; (2) a second-round questionnaire to deepen and clarify the salient issues emerging from Round 1 responses; and (3) a third-round questionnaire consisting of a few second-round questions that had not demonstrated consensus within the group, along with other questions to elicit more precise recommendations on how to address the pressing issues in POR. Throughout the study, both the broad term POR and the more focused term 'patient engagement in research' (PER) were used.

\section{Study panel}

Panelists were drawn from the pool of 25 trainees selected as part of the first cohort of awardees (of POR-specific scholarships, training and career awards) funded by a partnership between Fonds de la recherche du Québec-Santé (FRQS, provincial funding agency) and the Quebec SPOR SUPPORT Unit (hereafter, the Unit). This partnership aimed to create opportunities for establishing networks between experts working in this emerging field of study. Being a current awardee (i.e., graduate and postgraduate student at master, doctoral and postdoctoral levels, or a clinician-researcher in family medicine) of this partnership was the only inclusion criterion for participation in the present study. The objective was that all panelists were 
- 10 open-ended questions to generate the content of Rounds 2 and 3

- Main topics: ECRs' research experience with POR; POR definition, feasibility, utility, contribution; patients' roles and contributions;

Round 1 (n=18)

April 7 to

May 162016 ECRs perceived barriers and facilitators of doing POR; ECRs' needs

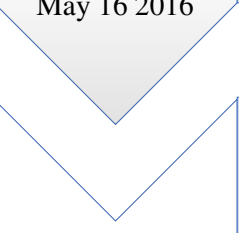

Round 2 (n=18) June 27 to August 162016

- 31 questions (29 closed questions and two optional open-ended questions) - Main topics: POR novelty; POR definition and values; patients' roles and contributions; ECRs' perceived barriers and facilitators (challenges); resources needed; ECRs recommendations; level of preparedness in doing POR; anticipated future of POR

- 27 questions (17 closed questions; four mandatory and six optional openended questions)

- Main topics: POR novelty and definition; patients' characteristics; ECRs' perceived challenges in doing POR; resources needed from ECRs

Round 3 (n=16) September 30 to November 28 2016 to conduct efficiently POR projects; perceived roles and responsibilities of the key stakeholders

Fig. 1 The topics in bold pertain to the first objective; they are presented and discussed in this article

"early career researchers" (trainees and untenured researchers). This convenience sampling was used, because this was an opportunity to capture a variety of students and researchers known to be exposed to POR in a pool of awardees. The scientific director of the Unit sent an initial email announcing the coming research study. Afterwards, a researcher (SB) invited the awardees to participate.

The two principal investigators (PIs) of this project (GR, JCBP) were also panelists and main authors of this manuscript. All other panelists were invited to collaborate in the writing of this manuscript as co-authors only after having completed the third round, to avoid generating pressure or incentive to participate and thus causing bias. GR and JCBP took measures to minimize any forms of bias regarding their different roles in the project: they worked with a third researcher (SB) that was neutral in the research process. During the analysis, equal "weight" was given to all findings and SB had the role to make sure that no perspective was favored. Regular debriefing meetings were held to discuss and challenge the entirety of the research process.

Although Delphi surveys traditionally rely on experts in a given field, it is becoming increasingly accepted for them to be conducted among panelists with non-advanced and/ or technical expertise [33]. Delphi surveys may involve professional experts on a specific topic or issue $[34,35]$ or panelists whose expertise derives from practical experience and knowledge [33, 36], or various combinations of professional and experiential expertise. In a paradigm such as POR, it is of utmost importance to consider and value experiential knowledge. It was for this reason that ECRs were recruited as panelists in the present study, as they are the ones simultaneously developing both experiential and traditional academic/professional expertise and who are most expert on the subject of their experience with POR.

\section{Questionnaires for each round}

The first-round questionnaire, developed from a non-systematic literature review, including empirical studies and gray literature (e.g. NIHR, PCORI, CIHR, cited above), consisted of five questions eliciting basic demographic information, one question asking panelists to self-evaluate their level of knowledge of POR, and ten open-ended questions designed to both cover and identify specific issues relevant to the research objectives. Of the latter, three questions explored panelists' own experience with POR, two pertained to defining POR-i.e., their own definition and understanding of POR-and patients' place in it, and four dealt with POR feasibility, utility, and ECRs' future needs. The first round questionnaire aimed not only to elicit an overall perception of POR, but also to describe the challenges and issues felt and experienced by 
ECRs. Responses to these open-ended questions served to generate the remaining two rounds of the study.

The second round consisted of 31 questions, including 24 Likert-scale type questions, four questions in which panelists were asked to choose the most pertinent items from lists, one prospective question, and two optional open-ended questions.

The third round differed from a classical Delphi third round in that only four of its 18 questions were repeated exactly as presented in Round 2. For those questions, panelists had the opportunity to recalibrate their ratings after considering the group's anonymous summary ratings. Eleven questions took up where the second-round questions left off and sought to deepen the subject or refine the level of (dis)agreement. Three questions were novel to the third round but were based on panelists' second-round comments. For questions that presented second-round aggregate response data, graphical representations of response frequency and brief statistical summaries (means and standard deviations) were used. The questionnaires for all three rounds are presented in more detail [see Additional file 1].

As this study was conducted in Quebec, the questionnaires were administered in French and panelists could respond in French or in English; only one responded in English. As such, most statements quoted in this paper have been translated by the authors.

\section{Data analysis}

Round 1 qualitative analysis was conducted independently by three researchers (GR, JCBP, SB) using QDA Miner 4.1.27, NVivo Version 11, and a manual approach (using Excel) to manage the data. The data analysis process was inspired by the three generic phases of inductive content analysis [37]: preparing the data, organizing and reporting them. Firstly, researchers read all the qualitative material, line by line. They coded the meaningful textual segments. They regrouped and classified codes into larger categories. Discussions were held among the three researchers to compare codes and categories. Throughout the analysis, the researchers referred back to the questions and research objectives to highlight significant data. Broad categories emerging from the responses were identified in the first-round questionnaire, and issues were subsequently categorized thematically. The three researchers compared categories and issues classifications until consensus was achieved. Once redundancy was eliminated, an aggregated list of issues suggested by the panelists was generated, which made up the content of the second round. When possible, the panelists' first-round arguments were synthesized into concise statements, and panelists' agreement with these was gauged in the second round. Findings for each round were visually represented and described into table, figure and text.

The quantitative responses were analyzed using Microsoft Excel (2013) and IBM SPSS Statistics Version 22.0. All Likert scales consisted of seven points $(1=$ "completely disagree", 4 = "neither agree nor disagree", 7 = "completely agree"). The threshold for agreement on a statement was established when at least 9 panelists $($ half +1$)$ selected a negative (1 to 3 ) or positive (5 to 7 ) value. Quantitative results were reported as mean \pm their standard deviation; median, and interquartile range (IQR). Correlations between the collected variables were calculated using Pearson's correlation coefficient (Rho), with values ranging from 0 , for a null correlation, to $+/-1$ for a total positive or negative correlation; $p=0.05$ was taken as the threshold of statistical significance in all tests performed.

\section{Results}

In this section, panelists' characteristics are presented first, followed by the five main themes: 1) ECRs' knowledge of and experience with POR; 2) the definition of POR; 3) patients' roles and contributions; 4) the anticipated future of POR; and 5) ECRs' roles in POR development and deployment. These themes reflect the perspectives and understanding of ECRs regarding many subjects related to POR.

\section{Panelists' characteristics}

Of the 25 awardees invited to participate, 18 (72\%) completed the first and second rounds and of those, 16 of 18 (89\%) completed the third round. It means that 16 of the total population of 25 (64\%) completed the study. This final panel of 16 encompassed a variety of profiles. Most $(n=12)$ were at various stages of training (master, $\mathrm{PhD}$, postdoctoral fellowship), and four were clinicians (general practitioners). Panelists were from different disciplines involved in health sciences research. All 16 had bachelor's degrees, 15 had master's degrees, six were medical doctors (MDs), six were PhDs (two of whom were also MDs), and four had postdoctoral research experience. Table 1 presents panelists' demographic characteristics.

\section{Knowledge of and experience with POR}

Panelists' self-assessed knowledge level regarding POR fell into four categories: "becoming familiar with POR" $(n=4)$, "basic knowledge of POR" $(n=6)$, "advanced knowledge of POR" ( $n=5)$, and "mastery of POR" $(n=1)$. The latter (with mastery) explained: "I build my research projects in partnership with patients. Most of my collaborators are patients." (Panelist $\mathrm{C}$ )

Ten of the 16 panelists reported having engaged patients in research: two had engaged patients as early as the identification of research needs and writing the 
Table 1 Panelists' demographic characteristics

\begin{tabular}{|c|c|}
\hline Characteristics & Panelists $(n=16)$ \\
\hline \multicolumn{2}{|l|}{ Age (years) } \\
\hline$<25$ & 1 \\
\hline 25 to 34 & 7 \\
\hline 35 to 44 & 7 \\
\hline 45 to 54 & 1 \\
\hline \multicolumn{2}{|l|}{ Gender } \\
\hline Male & 7 \\
\hline Female & 9 \\
\hline \multicolumn{2}{|c|}{ Highest degree of education completed ${ }^{\text {a }}$} \\
\hline Bachelor's & 16 \\
\hline Master's & 15 \\
\hline MD (Medical doctor) & 6 \\
\hline Doctorate (PhD) & $6^{\mathrm{b}}$ \\
\hline Postdoctoral studies & 4 \\
\hline \multicolumn{2}{|l|}{ Discipline studied } \\
\hline Medicine & 5 \\
\hline Pharmacology & 2 \\
\hline Public health & 2 \\
\hline Health sciences & 2 \\
\hline Nursing & 1 \\
\hline Clinical research & 1 \\
\hline Chemistry & 1 \\
\hline Epidemiology & 1 \\
\hline Bioethics & 1 \\
\hline \multicolumn{2}{|l|}{ Main occupation } \\
\hline Clinician & $2^{c}$ \\
\hline Master's student & 1 \\
\hline PhD student & 8 \\
\hline Postdoctoral fellow & 3 \\
\hline Professor & 2 \\
\hline Research professional & 1 \\
\hline
\end{tabular}

${ }^{\mathrm{a} S o m e}$ have more than one degree

${ }^{\mathrm{b}}$ Two of these six are also MDs

'They are two physicians whose time was divided between clinical, teaching, and research activities

research proposal and ending with data analysis. Choice of methodology and recruitment of study participants were also activities in which patients were involved. Reasons given for not engaging patients were POR being discussed too late in the research process and lack of resources, especially funding. Two panelists without practical experience with POR nevertheless claimed to have theoretical/conceptual knowledge.

Panelists' research experience with POR was moderately correlated with their main occupation, i.e., clinician or student (Rho $=0.552, p=.027$ ), and strongly correlated with self-reported knowledge level (Rho $=0.763, p=.001$ ).
However, there was no statistically significant relationship between panelists' level of formal education and their self-reported knowledge level $(p=0.281)$.

\section{Definition of POR}

Panelists were asked first to define qualitatively their conception of POR. That exercise produced 14 statements/propositions, from which panelists subsequently chose those they deemed most appropriate. Panelists agreed $^{1}$ on a broad, five-pronged definition of POR (hereafter called "defining elements"): 1) valuing, mobilizing, and legitimizing the experiential knowledge of patients living with a particular health condition; 2) conducting research that focuses on patients' concerns, participation, and outcomes; 3 ) integrating active partnership among a variety of actors (researchers, clinicians, decision-makers, institutions, patients, families, etc.); 4) conducting research with patients, for patients, and not only on patients; and 5) informing research with patients' viewpoints to improve healthcare, health research, and (patients') health knowledge. Furthermore, panelists concurred that "the time has come for the community of practice to agree on a definitional framework [of POR], and particularly on patients' degree of engagement, their roles, and mechanisms allowing their involvement" (5.69 $\pm 1.49 ; 6, \mathrm{IQR}=2)$.

Panelists identified 19 values they perceived to be fundamental to POR. Most relate to the nature of the relationship (e.g. collaboration, participation, communication, trust), the egalitarian aspect of POR (e.g. power sharing, self-fulfillment of peers on an equal footing, transparency), and inclusivity (e.g. open-mindedness and inclusiveness).

In the first round, panelists responded in their own words on their views regarding the purposes, utility, and benefits of POR. Their responses were then grouped into 14 propositions, and all panelists were asked to select five to eight of those that were most significant for them (Fig. 2). Figure 2 presents means of all the panelists. They agreed strongly on three propositions in which POR made it possible: 1) to better understand patients and their opinions and/or care and services received; 2) to integrate patients into the complex research process; and 3) to challenge existing knowledge that is taken for granted and to develop new knowledge based on the shared perspectives of researchers and patients.

Panelists also believed integrating POR into their research projects constituted an opportunity for them, both scientifically and academically $(5.38 \pm 1.26 ; 5, \mathrm{IQR}=2)$, in terms of publications, funding, and curriculum vitae (CV).

Panelists agreed moderately that POR is a novel approach to conducting research $(4.81 \pm 1.72 ; 5, \mathrm{IQR}=2)$. Similarly, they positioned the concept of POR as only slightly closer to innovative than recycled $(4.44 \pm 1.72 ; 5$, $\mathrm{IQR}=1$ ). Panelist $\mathrm{N}$ reflected the aggregate view that the 


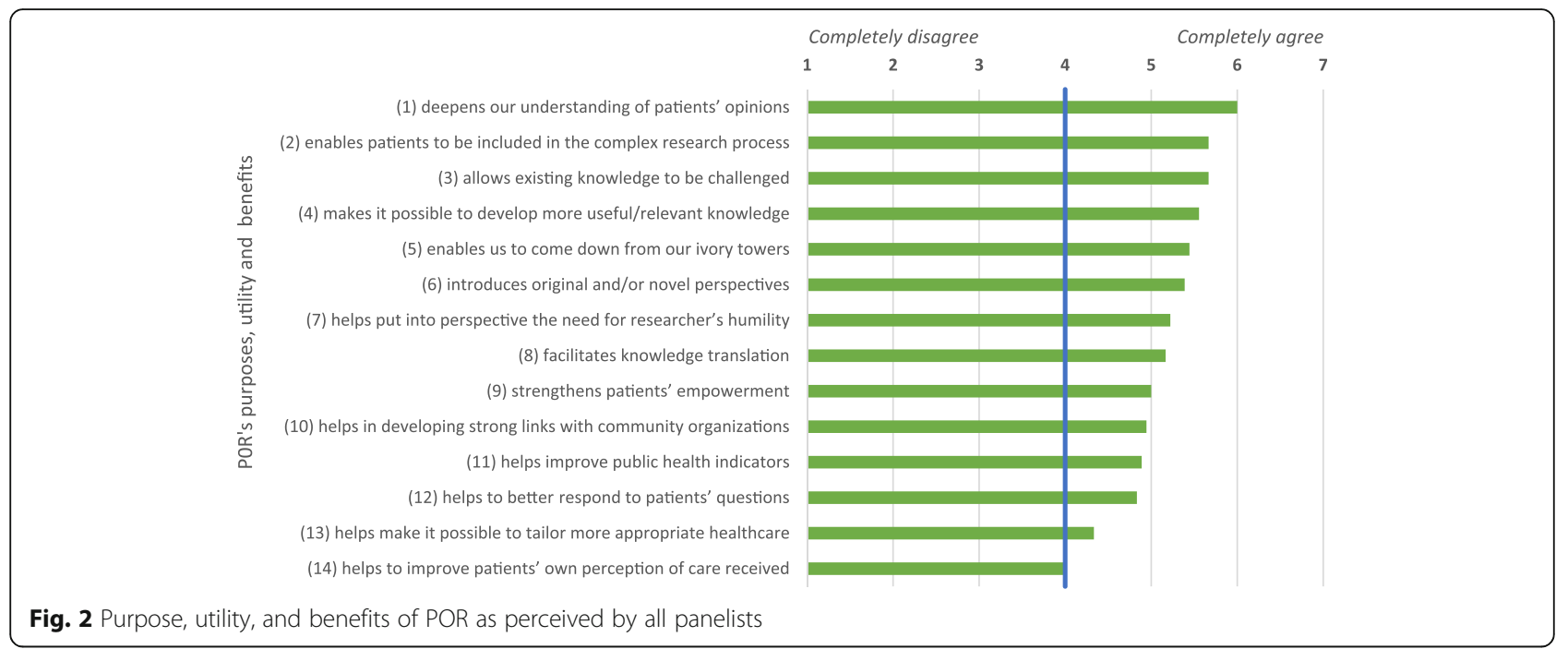

concept is simultaneously "new and recycled". Others, such as Panelist $\mathrm{O}$, cautioned against mistaking POR's recent trendiness for novelty. That panelist did not consider POR to be novel approach, as illustrated in the following excerpt:

"Patient-oriented research has always existed. I think there's a danger of confusing the reality of research with categories established by funding agencies. It's useful for the CIHR to create a new award category grouping a whole series of research themes that were previously distributed across many distinct labels." (Panelist O)

\section{Patients' roles and contributions Level of patient engagement}

Panelists suggested many terms for patients engaged in research: patient-collaborator, patient integrated/engaged/ involved in research, patient-educator, patient-researcher, peer research assistant, and patient-partner in research. In the present study, in the context of POR, the most commonly (7/16) preferred term was patient-partner in research (PPR). One panelist cautioned that it would be best not to rely on the term patient at all, as it might reduce individuals to their health condition and might inadvertently exclude the family from the partnership.

The panelists' preferred terminology, PPR, was aligned with their perceptions of patients' roles within the research team. Panelists considered PPRs to be integral members of the research team $(5.31 \pm 1.66 ; 6, \mathrm{IQR}=2)$, but were less convinced that PPRs should be considered co-researchers $(4.50 \pm 1.75 ; 5, \quad$ IQR $=3.5)$ who would contribute to all decisions throughout the research process. Rather, they were more in favour $(5.19 \pm 1.64 ; 5$, $\mathrm{IQR}=3.5$ ) of attributing to PPRs an advisory role, in which they could be called upon to voice opinions and to participate in certain decisions made over the course of the research process.

Panelists ascribed roles to PPRs throughout the research process, i.e., from the early to the late stages of a project, especially in these activities: identification of research needs, knowledge translation, research impact evaluation, and recruitment of participants. Panelists were less convinced about involving PPRs in choosing methodologies and in drafting grant applications.

Figure 3 shows the panelists' mean levels of agreement on the suitability of involving PPRs in each stage of the research process.

\section{Feasibility of POR in all types of research}

Besides considering the specific research activities that were more appropriate for PPR involvement, panelists also considered what types of research were best suited for POR. They did not agree regarding the feasibility of engaging patients in all types of research: seven affirmed its feasibility, five unambiguously stated it to be unfeasible, while four others had more nuanced positions. Some types of science (e.g. basic science), research objectives, topics, and types of data collected (e.g. administrative) were deemed less suitable for POR than others:

"I believe it is feasible to involve patients in all types of research. There are, however, studies in which active patient involvement would not necessarily be essential. For example, a validation study of a measurement instrument would not necessitate involving patients at all stages of the study. (Panelist A)"

Otherwise, panelists believed $(5.56 \pm 1.63 ; 6, \mathrm{IQR}=2)$ it is reasonable to expect a research team, at the outset of 


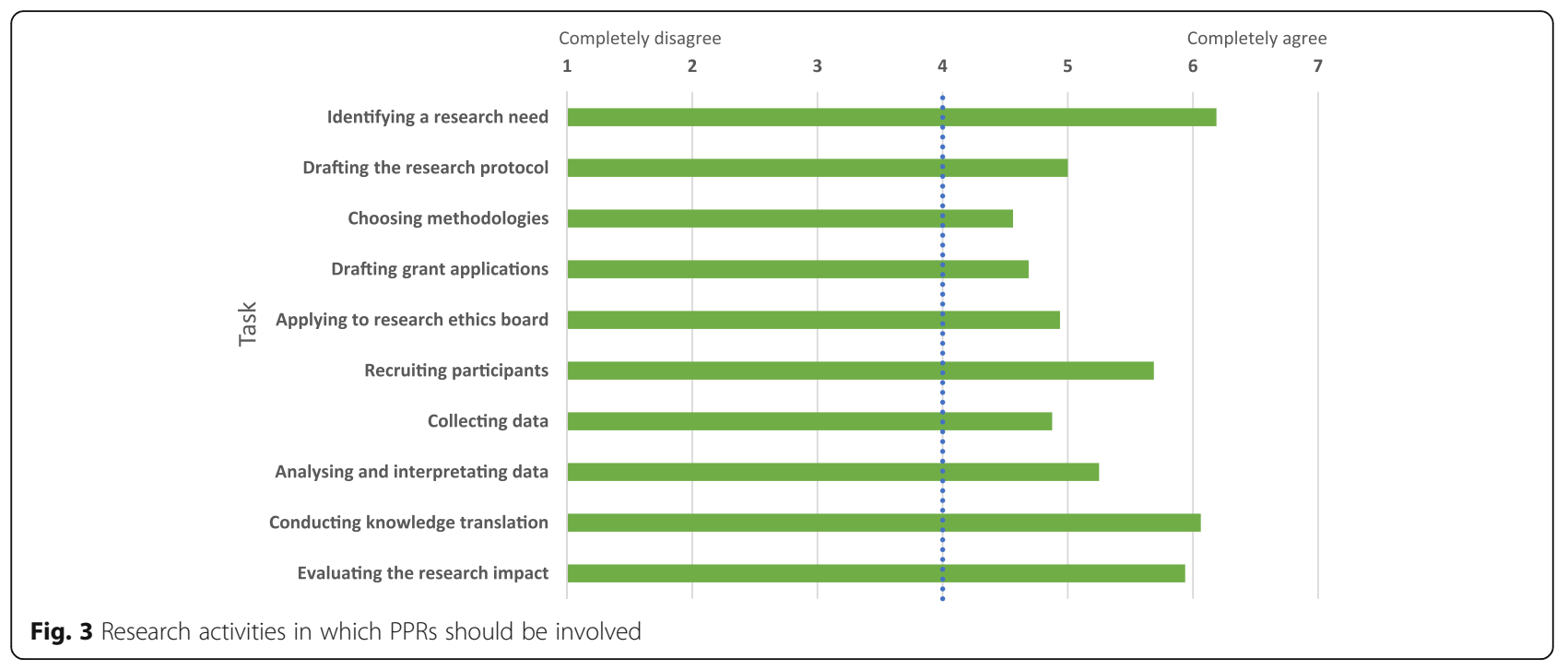

a POR project, to agree on the nature of each actor's (researchers, patients, collaborators, knowledge users) involvement.

\section{Desirable characteristics of PPRs}

Panelists disagreed $(2.81 \pm 1.72 ; 3, \mathrm{IQR}=3)$ that all patients, regardless of profile, were equally suited for inclusion in a research project. In other words, panelists believed there are optimal profiles for patients involved in POR. Figure 4 displays panelists' level of agreement regarding the desirability of various characteristics when recruiting PPRs. The most desired characteristics related mainly to patients' willingness to get involved in research, curiosity, interest in POR, absence of conflict of interest, and ability to interact with the research team.

\section{Anticipated future of POR: from uncharted waters to mainstream practice}

Panelists projected that POR will become mainstream in health research in 6.5 years (median value, in 2022-2023) (final IQR = 5.5; second-round $I Q R=5$ ), with responses (over all rounds) ranging from 1 to 15 years. Table 2 shows panelists' rationales for their projections.

Most panelists perceived POR and its future optimistically, with statements such as: "...a promising approach that will inspire many research disciplines"; "POR will be increasingly known and used"; "it will be further integrated into research projects and taught in postgraduate programs"; "its positive impacts have already been demonstrated and will continue to spread."

However, some panelists felt POR is an "evolving concept" whose definition remains to be refined. Those panelists, whose position was more nuanced, believed in POR's benefits and impact, while at the same time acknowledging its challenges. They highlighted some resources needed to ensure the sustainability of POR, such as collective mobilization of a variety of stakeholders, training, funding, and so on.

"I view the future of POR with a certain optimism, in that POR has been shown to have positive impacts, such as on health services and population health, among others. However, as a society, we need to go beyond just believing in the merits of such an approach. We need to actually deploy resources and put in place the winning conditions to support such an approach, and this will require a commitment from a variety of actors, organizations, networks, and funders to ensure sustainability. Therefore, a collective effort will have to be made to ensure the viability of such an approach. In this sense, the future of POR is promising but presents significant challenges. (Panelist L)"

Other panelists were cautious about the future of POR because they thought resources were lacking to ensure its survival and sustainability.

\section{ECRs' roles in POR development and deployment}

In the second round, panelists were asked to respond to the open-ended question: How do you see ECRs' roles in the development of POR and its deployment? This question was directly related to one of the research objectives. The reported ECRs' roles were related to one or more of the following: 1) their relationship with POR (including a commitment to better understand this new research paradigm and to engage PPRs in their project); 2) their own personal development based on the characteristics of POR (e.g. inclusive, open-minded); 3) the adoption of a reflexive and critical posture towards the approach, particularly regarding POR's ethical aspects; and 4) active engagement in POR development, awareness-raising, and dissemination. Although all these roles may not necessarily 


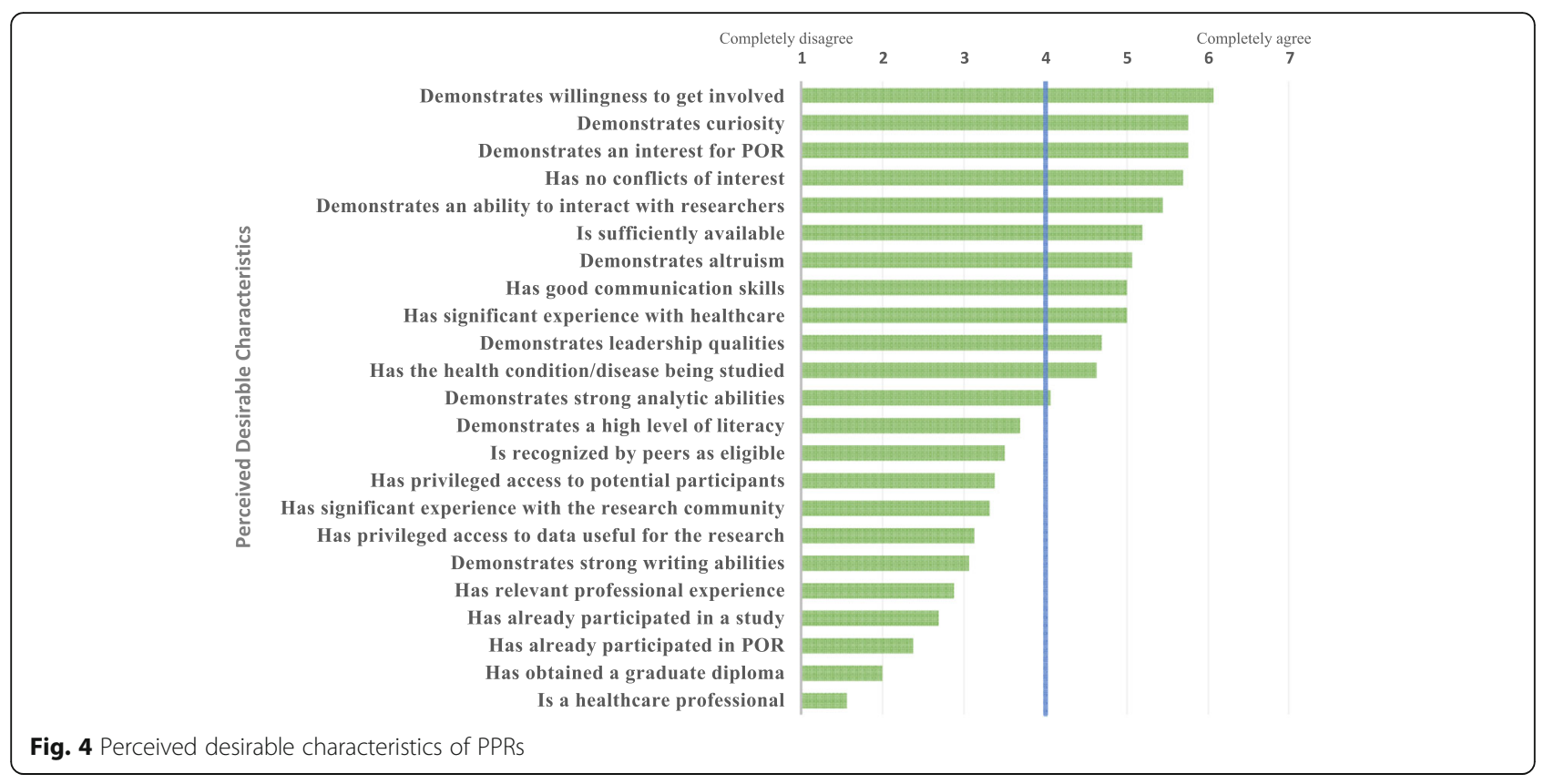

have to be assumed by every researcher, ECRs as a community were seen as instrumental to POR in the four above-mentioned manners.

\section{Discussion}

\section{Main findings}

This study aimed to present empirical evidence of ECRs' perspectives on the definition of POR, patients' roles in research, the anticipated future of POR, and, above all, the roles ECRs could play in developing and deploying it.

The majority of panelists self-reported having minimal or basic knowledge of POR. Some saw it as an evolving concept. Panelists expressed the need for a definitional framework of POR that would set out patients' degree of engagement, their roles, and how they should be involved.
For most of the ECRs, the definition of POR was not entirely clear, even though they agreed on certain defining elements drawn from the five-pronged definition on which almost all the panelists agreed. These defining elements were: patients' experiential knowledge; research with patients, focusing on patients' concerns, participation, and health outcomes; research informed by patients' viewpoints to improve healthcare, health research, and (patients') health knowledge; and active partnership among a variety of actors.

Regarding patients' roles and contributions, panelists positioned themselves on three aspects: the perceived role of PPRs, the research activities in which they would be involved, and PPRs' desirable profiles. They agreed on the inclusion of PPRs more as advisors than as co-researchers.

Table 2 ECRs' projections regarding POR's becoming mainstream

\begin{tabular}{|c|c|}
\hline Time before POR becomes mainstream & Rationales \\
\hline 0 & Already mainstream, but for a limited community \\
\hline 1 to 5 years & $\begin{array}{l}\text { Already mainstream } \\
\text { Unknown and underused approach } \\
\text { Increasingly used and relatively easy to integrate into research if funding is available } \\
\text { Efforts have been made and must continue to be made to make it mainstream } \\
\text { Promising solution for knowledge translation } \\
\text { Time needed for culture and paradigm changes }\end{array}$ \\
\hline 6 to 10 years & $\begin{array}{l}\text { Important changes required at multiple levels (micro, meso, and macro), including } \\
\text { additional human and financial resources } \\
\text { Evidence needs to be published demonstrating positive results and examples of } \\
\text { fruitful POR, but it takes time to get these positive results } \\
\text { Need to wait for the next generation of researchers and government funding } \\
\text { Potential need to integrate POR into curricula/programs and address the issue of } \\
\text { professionalization of PPRs }\end{array}$ \\
\hline
\end{tabular}


They considered the most desirable characteristics of PPRs to be: willingness, curiosity, interest in POR, no conflict of interest, and capacity to interact and communicate with researchers. Interestingly, the least desired characteristics were those that tended towards having a professional background or experience as a research participant.

Regarding the anticipated future of POR, perceived by the majority of ECRs with optimism, but with skepticism by others, panelists perceived POR to be likely to become mainstream in about seven years (i.e., around 2023). This means that current and prospective ECRs would be at the forefront of this paradigm shift in research. Panelists believed they had a role in developing and deploying POR, for example, by being knowledgeable about it, being predisposed towards it (maintaining supportive attitudes and characteristics), adopting a critical and reflexive posture, and being actively involved.

\section{Knowledge of POR, its definition and perceived benefits}

The defining elements of POR, as perceived by ECRs, reflected a way of doing research that is value-laden, i.e., conducted in partnership with patients and various actors. POR is built on recognizing and valuing experiential knowledge and integrating it into other ways of knowing, with the aim of informing scientific activity and carrying out the mission of research by producing relevant outcomes. Its defining elements, from the panelists' standpoint, offer an interdisciplinary perspectiveapplicable to a variety of health domains, disciplines, and sectors-and are not centred on one specialized methodology for conducting POR. Within the POR approach, many terms remain to be defined, as evidenced by the lack of consensus in the literature on the definitions of terms such as 'patient' and 'engagement', noted in a recent review paper [7]. In this study, ECRs considered 'patient-partner in research' (PPR) to be the most appropriate term for referring to patients participating in POR.

With their agreement on defining elements, panelists acknowledged the important socio-scientific benefits of conducting POR, such as integrating patients into research, challenging existing knowledge that is taken for granted, and developing new knowledge based on the shared perspectives of researchers and patients. Panelists also perceived the potential personal benefits conducting POR can generate for them. Given that ECRs are generally on the lookout for opportunities for career advancement [24], in order for POR to be appealing, it has to be seen to offer certain professional advantages to compensate for its time-intensive nature. Becoming experts in POR has the potential to position ECRs as innovators in developing this particular area of health research. Even if the benefits of involving PPRs on the researchers themselves have been documented elsewhere [19, 38] (e.g. acquiring new knowledge and skills, seeing the patients differently, changing the way of doing research), the perspective of POR as a career opportunity is novel to the present study.

\section{Patients' roles and contributions}

ECRs' ideas about which research activities are more suitable for PPR involvement differ slightly from those found in a systematic review by Domecq Grace et al. [16], where a higher number of studies documented patients' contributions in agenda setting, study design, recruitment, and implementation. Patients were engaged more commonly in the early stages of research and less in the execution and translation stages (except for implementation). In the present study, the ECRs shared an overall high level of agreement that all research activities are suitable for involving PPRs; all activities were rated above 4 , the neutral value. Some were rated higher than others; for instance, the identification of research needs and knowledge translation were rated above 6 out of 7 (close to "completely agree") and some others were rated lower, such as engaging PPRs in the choice of methodologies and the writing of the study protocol. It would be interesting, in future research, to deepen the question of research activity suitability for PPRs and to explore ECRs' perspectives and compare them with those of other POR stakeholders. For example, what does it mean for ECRs to integrate patients in all or specific research activities? Is there an issue of preserving one's own socio-professional identity and maintaining a monopoly on specific research activities?

While acknowledging that not just any patient could be recruited for POR, the panelists were in agreement that some characteristics were more desirable in PPRs than others. The most desirable were a personal predisposition to POR and genuine engagement (e.g. willingness, curiosity, interest, no conflict of interest), as well as good interpersonal skills (e.g. ability to interact and communicate effectively with the research team), followed by the experience of having received healthcare and living with a medical condition. Interestingly, the less desired characteristics were those that tended towards having professional qualities and status, as well as prior experience with research. However, some studies [39-43] embraced the vision of developing and defining a taxonomy of patients' competencies that would enable them to contribute optimally as partners by mobilizing their experiential knowledge in their care (as patient-resources), in research (as patient-researchers) and in healthcare education (as patient-trainers). This idea of patients' competencies acknowledges not only PPRs' experience-based knowledge of living with a disease, but also their capacities to mobilize it skillfully through their engagement in care, research, and education-something not raised by the panelists. It is conceivable that the ECRs 
were not aware of these PPR competencies. Thus, the main observation that can be made in comparing this study and competencies-oriented studies is that there appears to be a gap between the desirable characteristics of PPRs and a patient-based competency approach fostering the mobilization of experiential knowledge. While it is not yet clear how PPRs' characteristics and competencies should best be reconciled to fully appreciate their contribution as well as to recruit and involve them optimally in research, there is probably fertile ground here to train non-expert trainees in POR on these questions.

\section{Panelists' level of knowledge}

Despite the momentum building up around POR (e.g. scholarship opportunities, research funding, emergence of specific training) and the fact that the panelists were all recipients of competitive POR scholarships, the majority of panelists self-reported as having minimal or basic knowledge of POR. Their level of knowledge was surveyed only in the first round, whereas Rounds 2 and 3 took place two and five months later, respectively. It is conceivable that the ECRs' level of knowledge would have been different if evaluated at Round 3. In fact, the panelists received training and gained experience with POR through their individual research projects and collaborations, including an intensive in-person training session between Rounds 1 and 3. Over the course of the study, they had opportunities to engage in specialized POR networking as part of their scholarship, which could have modulated their knowledge and understanding of POR.

According to the ECRs, POR would benefit from greater recognition, clearer definition, wider dissemination, and increased funding. ECRs would need a definitional framework, perhaps due to their unease at finding themselves in a context without normative and operational guidance to support them in understanding POR and how to conduct it adequately. However, feeling the need for a definitional framework does not mean resources do not exist. ECRs' perspectives on POR must be interpreted with caution due to their evolving familiarization with and learning regarding this approach. ECRs are experts on their own situation, but not necessarily experts on POR itself.

One of the main implications for ECRs is that their development as independent researchers is all the more marked by challenges. One reason for this might be that support networks are still embryonic and the POR research field is not as well known as others (which can complicate job searching). Then, applying POR can be complex for junior researchers; considerable time and resources are required to conduct POR diligently, authentically, and effectively. There is therefore a clear need for a coherent and concerted strategy for POR capacity development, in which the perspectives and specific needs of ECRs are taken into account.

\section{Anticipated future of POR}

If, as many panelists projected, POR is likely to become mainstream by about 2023, then current and prospective ECRs will be at the forefront of this paradigm shift in research. Some panelists anticipated a positive future for it, albeit one requiring some preparation on the part of the research community, funders, and universities. It will take time and effort to put in place the human and material resources, as well as the training, needed to embed POR in research and ensure its long-term sustainability. It is conceivable that the perceived "non-mainstream" nature of POR might be seen as a competitive advantage to enter the market (i.e., to conduct research in the field of POR) and to create a strong POR identity. It is possible that, in the future, the market could become increasingly competitive and saturated, as more researchers apply for funding opportunities and funding success rates decelerate (although, once mature, the funding for this field should potentially be more substantial). In this anticipated future, ECRs believe they should play an active role in developing and implementing POR, as well as in facilitating consensus on a definitional framework. Such efforts are likely to produce significant results, considering that ECRs are the new and biggest wave of researchers [24] with a potentially strong role in bringing POR into the mainstream.

\section{ECRs' roles in developing and deploying POR}

Starting from the general description of roles (Results section), ECRs' roles were then grouped together and names assigned, to deepen the interpretation. This new organization of roles, as presented in Table 3, was inspired by the work of Crocker et al. [44], who summarized the perceived roles and impact mechanisms of public and patient involvement (PPI) contributors (patients, caregivers, and members of the public). Even though some of the perceived roles in that source and in this present study are different, such as "expert in lived experience", Crocker et al.'s [44] model offers a useful lens through which to view and interpret the results of the present study. In applying it to the findings of the present study, ECRs were found to play up to 10 roles, each having a different impact either on POR development and uptake, on their research community and environment, on patient involvement, on knowledge translation, and so on. It is not expected that every ECR would play all these roles. Rather, through their combined activities as a whole, this next generation of researchers will foster and broaden the networks of researchers interested and involved in POR, be active disseminators of POR, apply it genuinely (avoiding tokenism), and document its impact. 
Table 3 ECRs' potential roles in POR development and implementation

\begin{tabular}{ll}
\hline Role & Action mechanisms (impact) \\
\hline Knower & Being informed and knowledgeable about POR. Participating in meetings to \\
& provide informed opinions and present constructive criticisms. \\
Awareness-raising vector / champion & Informing and sensitizing other students, colleagues, research supervisors, and \\
& established or future researchers about the existence and potential benefits of \\
& POR while maintaining a critical perspective on this approach. \\
& Reflecting on POR through an ethical lens, e.g. proposing action to understand \\
Ethically sensitive thinker & how to conduct POR responsibly and sensitizing the research community regarding \\
& the responsibilities inherent in this approach. \\
& Disseminating reflections, definitions of engagement, practical experiences, and \\
kisseminator / broker & publications and communications. \\
& Encouraging the research community, peers, and colleagues to engage in POR \\
Motivator & Forming a community of practice to create spaces for reflecting on POR; building a \\
Builder / creator & network to guide new research and to train and support those interested in POR. \\
Teacher & Training and teaching students about POR. \\
Doer & Engaging patients in research, collaborating in POR projects. \\
Delegate / representative & Serving as a representative voice on committees (e.g. SPOR SUPPORT units, funding \\
& organizations, research committees). Participating in decision-making regarding the \\
& general directions of research (e.g. how to make research findings applicable and useful for patients). \\
Activist & Defending POR tenets and being involved in networks promoting POR.
\end{tabular}

Table 4 shows the summarized key findings of the study, its main takeaway messages. These key findings relate to the roles ECRs have to play in the future of POR as well as to insights into POR capacity development strategies.

\section{Strengths, limitations, and special considerations of the study}

One strength of the present study was that the panel was drawn from ECRs associated with the Quebec SPOR SUPPORT Unit, a Canadian population that has not been studied extensively in relation to POR. The sample's heterogeneity in terms of stage of training, main occupation,

Table 4 Takeaway messages from synthesized findings

\footnotetext{
- Even if ECRs are awardees and are part of an established POR network, a majority self-reported having minimal or basic knowledge of POR. There is therefore a clear need for a coherent and concerted strategy for POR capacity development, in which the perspectives and specific needs of ECRs are taken into account.

- ECRs had a slightly different understanding of patient engagement than those found in the literature. To make sure that we all have a common understanding of POR, ECRs expressed the need for a definitional framework of POR that would set out patients' degree of engagement, their roles, and how they should be involved.

- ECRs assigned different roles to PPRs in various phases of a research project. ECRs envisioned PPRs' desirable characteristics as genuine engagement, interpersonal skills, and experience with the health condition. Professionalization (i.e., having a professional background) was not seen as desirable.

- ECRs anticipate POR to become mainstream around 2023.

- ECRs are at the forefront of this paradigm shift in research and are willing to play many roles (up to ten) in POR deployment: knower, awareness-raising vector, ethically sensitive thinker, disseminator/ broker, motivator, builder/creator, teacher, doer, delegate/representative and activist.
}

and discipline represents a strength, providing insights from various profiles of students and researchers/clinicians. The findings of this study could potentially be transferable to other ECRs funded by comparable scholarships in other Canadian provinces, because they are from the same jurisdiction and funded under the same research fund. However, considering the atypical nature of these ECRs (i.e., they received competitive awards and they are integrated into a POR network), the study design and small sample size, the results are not generalizable.

ECRs' perspectives should not be interpreted as claiming to describe and crystallize a certain vision of POR as true, but rather, should be seen as informative about how the future generation of researchers envision POR and what developments they expect or desire. Their perspectives are coloured by their experience with and knowledge of POR, their occupational backgrounds, and their personal ways of seeing the world (i.e., epistemological and ontological stances). Consequently, their (mis)understandings of POR and its components may help to uncover the gap between what ECRs know, do, and believe, on the one hand, and the current state of knowledge on POR, on the other. Ideally, resources (such as training) should be deployed that not only take into account ECRs' perspectives, but also support them in understanding POR's foundations and conducting it according to best practices.

A special consideration may be that the panelists were all nurtured within a certain environment, that is, the Quebec SPOR SUPPORT Unit, which has a comprehensive role and view of POR that may differ substantially 
from those of INVOLVE or PCORI. Therefore, panelists' specific understandings of POR may have been influenced by this perspective. Among the panelists, two (GR \& JCBP) were also PIs of the Delphi study. A third researcher (SB) served as study monitor throughout the process to minimize any potential bias that might arise from having panelists as PIs and to ensure responses remained completely anonymous to the PIs.

Although reporting means and standard deviations, alongside median and inter-quartile range, may lead to a misinterpretation of our results as if they were derived from a large sample, they have nonetheless been included as providing more detailed information. Statistical hypothesis testing was never intended in this study, and descriptive statistics are intended as supplementary to the qualitative results. Considering that the second and third rounds were constructed iteratively based on the preceding ones, and that the first round collected qualitative data to inform the two quantitative rounds, the results can be said to have emerged almost exclusively from the panelists. Except for specific points required to satisfy the research objectives, the contents of second- and third-round questionnaires were panelist-generated. The fact that most Round 3 questions were new was double-edged: this design allowed the Delphi process to cover a range of topics regarding ECRs' perspectives on POR, but it meant some subjects appearing for the first time in Round 3 were not then subjected to further iterative controlled group feedback to panelists.

\section{Avenues for further research}

This study targeted only ECRs' perspectives. Future research with patients would be complementary to contrast and confirm this study's findings in terms of how POR is defined, PPRs' roles and contributions, their level of engagement, their most suitable characteristics, the envisioned future of POR, and PPRs' role in developing and implementing POR. For instance, would patients agree with ECRs that their involvement is most appropriately confined to specific research activities? Would patients see a role for themselves in the other stages of a project? If so, what implications might such a role have in terms of the issue of professionalization of patients?

\section{Conclusions}

This study sheds light on how ECRs perceive POR: its definition, patients' roles and contributions, the future of POR, and their roles as ECRs in developing POR. It is conceivable that these perceptions can shape their current and future practice of POR. These findings, complementing the existing literature, map out the many areas where capacity development can be supported and strengthened. In the view of most of the panelists, POR needs a definitional framework, and the broad question of patients' roles and contributions has to be addressed. The panelists recognized that POR is important and brings clear benefits to research, as well as potential opportunities for career advancement. The majority were optimistic regarding POR's anticipated future, albeit with a few dissenting skeptical voices. They envisioned that the approach would become a mainstream practice in about 2023. Many stated that time is needed for cultural and paradigm changes, and resources have to be injected to support these changes. This highlights the need to better support ECRs in their training and development as independent researchers. This would put current and prospective ECRs at the forefront of this paradigm shift in research, and those ECRs could then be in a strategic position to contribute actively and positively to POR development and deployment. Whether felt particularly by ECRs or all the actors involved, there is a clear need for a coherent and concerted strategy for POR capacity development, taking ECRs' perspectives and specific needs into account. It is essential to ensure that the next generation of researchers are nurtured and empowered to reach their full potential, which is a promising safeguard for supporting effective research and constant development of POR from the very beginning of researchers' careers.

\section{Endnotes}

${ }^{1}$ When asked in the third round to select two statements/propositions, 30 of the 32 choices were among these five reported statements/propositions.

\section{Additional file}

Additional file 1: Questionnaires for all three rounds (Rouleau, BélislePipon et al.). (PDF $546 \mathrm{~kb}$ )

\section{Abbreviations}

CIHR: Canadian Institutes of Health Research; ECRs: Early career researchers; IQR: Interquartile range; M: Mean; NIHR: National Institute for Health Research; PER: Patient engagement in research; PI: Principal investigator; PICORI: Patient-Centered Outcomes Research Institute; POR: Patient-oriented research; PPR: Patient partners in research; SPOR: Strategy for PatientOriented Research; SUPPORT: Support for People and Patient-Oriented Research and Trials

\section{Acknowledgements}

A special thanks to Marie-Dominique Beaulieu for having supported this project and for her careful review of this manuscript. We would like to acknowledge the contribution of Donna Riley in editing and preparing the final manuscript. Thanks also to those who participated in the study without completing all three rounds.

\section{Funding}

This work was funded by the Quebec SPOR SUPPORT Unit (SU1 - 139759). The funding agency had no role in the conduct of the research. The authors had full freedom to write about SPOR, POR, and Québec SPOR SUPPORT

Unit without any pressure.

\section{Availability of data and materials}

The datasets used and/or analyzed in the current study are available from the corresponding author on reasonable request. 


\section{Authors' contributions}

JCBP and GR conceived the study, participated in its design, coordination, and analysis, and drafted the manuscript. SB assisted in acquiring, analyzing and interpreting data and in drafting the article. PK and NF reviewed the initial protocol and supported the early phases of the project. All other authors cited in this paper read and approved the final manuscript.

\section{Ethics approval and consent to participate}

Ethics approval was obtained through the Health Research Ethics Committee (CERES) at the University of Montreal (\#16-044-CERES-D).

\section{Consent for publication}

N/A

\section{Competing interests}

One author reports a conflict of interest: in 2015, Cynthia Khanji won a scholarship funded by Pfizer Canada Inc. and the Réseau québécois de recherche sur les medicaments, which allowed her to do an internship at Pfizer Canada Inc. during the summer of 2016. She has also been working part-time at Pfizer Canada Inc. since December 2016. All other authors declare they have no competing interests.

\section{Publisher's Note}

Springer Nature remains neutral with regard to jurisdictional claims in published maps and institutional affiliations.

\section{Author details \\ ${ }^{1}$ University of Montreal Hospital Research Centre, Montreal, Quebec H2X0A9, Canada. ${ }^{2}$ Faculty of Nursing, Laval University, Quebec, Canada. ${ }^{3}$ The Petrie-Flom Center for Health Law Policy, Biotechnology, and Bioethics, Harvard Law School, Cambridge, USA. ${ }^{4}$ Health Law Institute, Dalhousie University, Halifax, Canada. ${ }^{5}$ Bioethics Program, University of Montreal School of Public Health, Montreal, Canada. ${ }^{6}$ University of Montreal, Public Health Research Institute, Montreal, Canada. ${ }^{7}$ Faculty of Medicine, Departement of Family and Emergency Medicine, University of Montreal, Montreal, Canada. ${ }^{8}$ Faculty of Nursing, University of Montreal, Montreal, Canada. ${ }^{9}$ Canadian Agency for Drugs and Technologies in Health, Ottawa, Canada. \\ ${ }^{10}$ Department of Family Medicine, McGill University, Montreal, Canada. \\ ${ }^{11}$ University of Sherbrooke, Sherbrooke, Canada. ${ }^{12}$ Faculty of Medicine, Laval University, Quebec, Canada. ${ }^{13}$ Faculty of Medicine and Health Sciences, University of Sherbrooke, Longueuil, Canada. ${ }^{14}$ Faculty of Pharmacy, University of Montreal, Montreal, Canada. ${ }^{15}$ Faculty of Pharmacy, Laval University, Quebec, Canada. ${ }^{16}$ Faculty of Medicine, Laval University, Quebec, Canada. ${ }^{17}$ Department of Epidemiology, Biostatistics and Occupational Health, McGill University, Montreal, Canada.}

Received: 15 March 2018 Accepted: 31 August 2018

Published online: 09 October 2018

\section{References}

1. Canadian Institutes of Health Research. Strategy for Patient-Oriented Research - CIHR [Internet]. 2010. Available from: http://www.cihr-irsc.gc.ca/e/ 41204.html. Cited 26 Nov 2017.

2. Canadian Institutes of Health Research. Strategy for Patient-Oriented Research - Patient Engagement Framework [Internet]. Ottawa; 2014 Jul. Available from: http://www.cihr-irsc.gc.ca/e/48413.html\#a7

3. Johansson V. From subjects to experts - on the current transition of patient participation in research. Am J Bioeth. 2014;14:29-31.

4. Mayer M. Seeking what matters: patients as research partners. Patient. 2012;5:71.

5. Boivin A, Lehoux P, Lacombe R, Burgers J, Grol R. Involving patients in setting priorities for healthcare improvement: a cluster randomized trial. Implement Sci. 2014;9:24.

6. Sacristán JA, Aguarón A, Avendaño-Solá C, Garrido P, Carrión J, Gutiérrez A, et al. Patient involvement in clinical research: why, when, and how. Patient Prefer Adherence. 2016;10:631-40.

7. Duffett L. Patient engagement: what partnering with patient in research is all about. Thromb Res. 2016:150:113-20.

8. National Institute for Health Research. INVOLVE Supporting public involvement in NHS, public health and social care research [Internet]. 2018. Available from: http://www.invo.org.uk/ Cited 18 Aug 2018
9. Patient-Centered Outcomes Research Institute. Research funding [Internet] 2018. Available from: https://www.pcori.org/sites/default/files/PCORIResearch-Funding.pdf. Cited 5 Sept 2018.

10. Canadian Institutes of Health Research. Evaluation of the Strategy for Patient-Oriented Research - CIHR [Internet]. 2016. Available from: http:// www.cihr-irsc.gc.ca/e/49937.html. Cited 11 Dec 2017

11. Nicholas D. Early-career researchers plant one foot in the future. Research Europe. 2018;2:7-8.

12. Lough S. Need to define patient engagement in research. Can Med Assoc J. 2015;187:E385-6.

13. Marlett N, Shklarov S, Marshall D, Santana MJ, Wasylak T. Building new roles and relationships in research: a model of patient engagement research. Qual Life Res. 2015;24:1057-67.

14. Carman KL, Dardess P, Maurer M, Sofaer S, Adams K, Bechtel C, et al. Patient and family engagement: a framework for understanding the elements and developing interventions and policies. Health Aff (Millwood). 2013:32:223-31.

15. Carman $\mathrm{KL}$, Workman TA. Engaging patients and consumers in research evidence: applying the conceptual model of patient and family engagement. Patient Educ Couns. 2017;100:25-9.

16. Domecq Garces JP, Prutsky G, Elraiyah T, Wang Z, Nabhan M, Shippee N, et al. Patient engagement in research: a systematic review. BMC Health Serv Res. 2014;14:89.

17. Hardavella G, Bjerg A, Saad N, Jacinto T, Powell P. How to optimise patient and public involvement in your research: doing science. Breathe Sheff. 2015;11:223-7.

18. Brett J, Staniszewska S, Mockford C, Herron-Marx S, Hughes J, Tysall C, et al. Mapping the impact of patient and public involvement on health and social care research: a systematic review. Health expect Int J public Particip health care. Health Policy. 2014;17:637-50.

19. Brett J, Staniszewska S, Mockford C, Herron-Marx S, Hughes J, Tysall C, et al. A systematic review of the impact of patient and public involvement on service users, researchers and communities. Patient. 2014;7:387-95.

20. Gradinger F, Britten N, Wyatt K, Froggatt K, Gibson A, Jacoby A, et al. Values associated with public involvement in health and social care research: a narrative review. Health Expect. 2015;18:661-75.

21. Manafò E, Petermann L, Mason-Lai P, Vandall-Walker V. Patient engagement in Canada: a scoping review of the 'how' and 'what' of patient engagement in health research. Health Res Policy Syst. 2018;16:5.

22. Manafò E, Petermann L, Vandall-Walker V, Mason-Lai P. Patient and public engagement in priority setting: a systematic rapid review of the literature. PLoS One. 2018;13:e0193579.

23. Bosanquet A, Mailey A, Matthews KE, Lodge JM. Redefining "early career" in academia: a collective narrative approach. High Educ Res Dev. 2016;36:890-902.

24. Nicholas D, Watkinson A, Boukacem-Zeghmouri C, Rodríguez-Bravo B, Xu J, Abrizah A, et al. Early career researchers: scholarly behaviour and the prospect of change. Learn Publ. 2017:30:157-66.

25. Bélisle-Pipon J-C, Rouleau G, Birko S. Early-Career Researchers' Views on Ethical Dimensions of Patient Engagement in Research, BMC Med Ethics. 19: 21

26. Goodman CM. The Delphi technique: a critique. J Adv Nurs. 1987;12:729-34.

27. Dalkey NC. The Delphi method: An experimental study of group opinion [Internet]. Rand Corporation Santa Monica, CA; 1969. Available from: http:// www.rand.org/content/dam/rand/pubs/research_memoranda/RM5888/ RM5888.pdf. Cited 1 Apr 2014

28. Rayens MK, Hahn EJ. Building consensus using the policy Delphi method. Policy Polit Nurs Pract. 2000;1:308-15.

29. Critcher C, Gladstone B. Utilizing the Delphi technique in policy discussion: a case study of a privatized utility in Britain. Public Adm. 1998;76:431-49.

30. Hasson F, Keeney S, McKenna H. Research guidelines for the Delphi survey technique. J Adv Nurs. 2000:32:1008-15.

31. de Loë RC, Melnychuk N, Murray D, Plummer R. Advancing the state of policy Delphi practice: a systematic review evaluating methodological evolution, innovation, and opportunities. Technol Forecast Soc Change. 2016;104:78-88.

32. Boulkedid R, Abdoul H, Loustau M, Sibony O, Alberti C. Using and reporting the Delphi method for selecting healthcare quality indicators: a systematic review. PLoS One. 2011:6:e20476.

33. Roberts-Davis M, Read S. Clinical role clarification: using the Delphi method to establish similarities and differences between nurse practitioners and clinical nurse specialists. J Clin Nurs. 2001;10:33-43. 
34. Boynton LA. What we value: a Delphi study to identify key values that guide ethical decision-making in public relations. Public Relat Rev. 2006;32: 325-30.

35. Smart KM, Blake C, Staines A, Doody C. Clinical indicators of 'nociceptive', 'peripheral neuropathic' and 'central mechanisms' of musculoskeletal pain. A Delphi survey of expert clinicians. Man Ther. 2010;15:80-7.

36. Broomfield D, Humphris GM. Using the Delphi technique to identify the cancer education requirements of general practitioners. Med Educ. 2001;35: 928-37.

37. Elo S, Kyngäs H. The qualitative content analysis process. J Adv Nurs. 2008; 62:107-15.

38. Staley K, Abbey-Vital I, Nolan C. The impact of involvement on researchers: a learning experience. Res Involv Engagem. 2017;3:20.

39. Direction collaboration et partenariat patient [Office of Collaboration and Patient Partnership] (DCPP). Guide pratique. Recrutement des patients partenaires. [Practical guide. Recruitment of patients partners]. [Internet]. 2015. Available from: http://docplayer.fr/71714641-Recrutement-desguidepratique-patients-partenaires-document-produit-par-l-equipe-de-ladirectioncollaboration-et- partenariat-patient -dcpp.html. Cited 5 sept 2018.

40. Direction collaboration et partenariat patient [Office of Collaboration and Patient Partnership] (DCPP). Référentiel de compétences des patients [Patients' competencies repository]. 2015. Available from: http://cio. partenaires-desoins.ca/wp-content/uploads/2016/11/BAO_CSS3900_ Referentiel_competences_des_patients-21avril2015-vf.pdf2015. Cited 5 sept 2018.

41. Karazivan P, Dumez V, Flora L, Pomey M-P, Del Grande C, Ghadiri DP, et al. The patient-as-partner approach in health care: a conceptual framework for a necessary transition. Acad Med. 2015;90:437-41.

42. Flora L. Le patient formateur : élaboration théorique et pratique d'un nouveau métier de la santé [The patient as trainer of medical staff: theoretical and pratical development of a new job to health]. Thèse de doctorat en Sciences de l'éducation [Doctoral thesis in education sciences]. Paris, France: Université Vincennes Saint Denis - Paris 8, Q20 1147 campus Condorcet. 2012

43. Flora L. Un référentiel de compétences de patient : pourquoi faire ? Du savoir expérientiel des malades à un référentiel de compétences intégré : l'exemple du modèle de Montréal [Patients' competencies repository: why ? From patients' experience-based knowledge to an integrated patient' competencies repository: the example of the Montreal model]. Sarrebrücken, Germany: Presses Académiques Francophones. 2015.

44. Crocker JC, Boylan A-M, Bostock J, Locock L. Is it worth it? Patient and public views on the impact of their involvement in health research and its assessment: a UK-based qualitative interview study. Health Expect. 2016;20: $519-28$

Ready to submit your research? Choose BMC and benefit from:

- fast, convenient online submission

- thorough peer review by experienced researchers in your field

- rapid publication on acceptance

- support for research data, including large and complex data types

- gold Open Access which fosters wider collaboration and increased citations

- maximum visibility for your research: over $100 \mathrm{M}$ website views per year

At $\mathrm{BMC}$, research is always in progress.

Learn more biomedcentral.com/submissions 